\title{
Current localization and Joule self-heating effects in $\mathrm{Cr}$ doped $\mathrm{Nd}_{0.5} \mathrm{Ca}_{0.5} \mathrm{MnO}_{3}$ manganites
}

\author{
A. S. Carneiro and R. F. Jardim* \\ Instituto de Física, Universidade de São Paulo, CP 66318, 05315-970, São Paulo, SP, Brazil \\ F. C. Fonseca \\ Instituto de Pesquisas Energéticas e Nucleares, CP 11049, 05422-970, São Paulo, SP, Brazil
}

(Dated: February 18, 2017)

\begin{abstract}
The effects of dc excitation current on the current-voltage curves of polycrystalline samples of $\mathrm{Nd}_{0.5} \mathrm{Ca}_{0.5} \mathrm{Mn}_{0.96} \mathrm{Cr}_{0.04} \mathrm{O}_{3}$ were investigated. The experimental results show that an abrupt jump of the voltage is concomitant with a huge increase in the temperature of the sample. A simple model and estimates for Joule self-heating effects support the experimental data. Moreover, the data strongly suggest that both the current localization in the metallic paths and local Joule selfheating effects are essential ingredients to understand the current-induced phase transition in phaseseparated manganites.
\end{abstract}

PACS numbers: 75.47.Lx, 71.30.+h, 64.60.Ak

Keywords: manganites, phase separation, Joule self-heating, current localization

It has been reported that phase control in manganites can be achieved by both the appropriate chemical substitutions $\frac{1}{1}$ and by application of external stimuli such as pressure $P, \underline{2}$ magnetic field $H, \underline{1}$ high-power laser irradiation,,$\underline{\underline{3}}$ and electric fields $E \stackrel{\underline{4}}{\underline{\underline{4}}}$ However, while both $H$ and $P$ change the bulk properties of the colossal magnetoresistance (CMR) manganites, the influence of light, X-rays, and electric current $I$ may result in a rather localized phase transition ${ }^{\underline{5}}$ In particular, effects induced by applied $E$ have been studied in both the charge/orbital-ordered (CO-OO) and the ferromagneticmetallic (FMM) states in single crystals and polycrys-

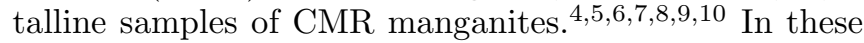
compounds, a drop in the electrical resistivity $\rho(T)$ above a threshold value of the applied dc $E$ (or dc current $I$ ) is generally observed ${ }^{6.7}$ This $E$-driven (or $I$-driven) insulator to metal transition $\mathrm{MI}$ is close related to the occurrence of a nonlinear conductivity $\stackrel{4.6}{ }$ The nonlinearity of the $I$ - $V$ curves, observed for $T<T_{C O}$, is thus explained by considering a percolation process due to the melting of the $\mathrm{CO}$ insulating (COI) phase into the metallic one. This interpretation is based on the opening of metallicfilament paths in a COI matrix. . $^{\text {5.6 }}$

Besides the common observation of nonlinearity in $I-V$ curves, this scenario is still not clear and other physical mechanisms have been proposed as the depinning of the $\mathrm{CO}-\mathrm{OO}$ state upon $E$ application ${ }^{7}$ the change in the orientation of the $\mathrm{OO}$ in the insulating state upon application of large $E \stackrel{10}{\longleftarrow}$ and an $E$-induced switching of the directional order of the OO states of $e_{g}$ electrons 11 Results of $I$ - $V$ curves under $H$ also indicated an increase of the magnetization at the same threshold $V$ where $I$ rises abruptly ${ }^{9}$ It was argued that Joule heating effects were insignificant and the formation of a less-resistive mixed state, but with higher magnetization than the initial CO

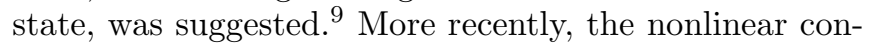
duction was described by considering a model for chargedensity-wave (CDW) motion. ${ }^{12}$
The proposed scenarios for the $I$ (or $E$ )-induced phase transition usually consider mechanisms related to the interplay between charge and spin degrees of freedom $7.8,9,10,11,12$ However, based on magneto-optical imaging of local magnetization and $I$ distribution, a different view has been recently proposed ${ }^{13}$ It was suggested that application of high density $I$ reduces the metallic channels due to local Joule self-heating effects. Increasing $I$ changes the metallic paths into insulating regions, further localizing the inhomogeneous current flow, and propagating the Joule self-heating. This process results in a rapid collapse of the metallic channels, which is related to an abrupt jump in the $I-V$ curves at a welldefined $I$ threshold $I_{T} \stackrel{13}{\underline{13}}$

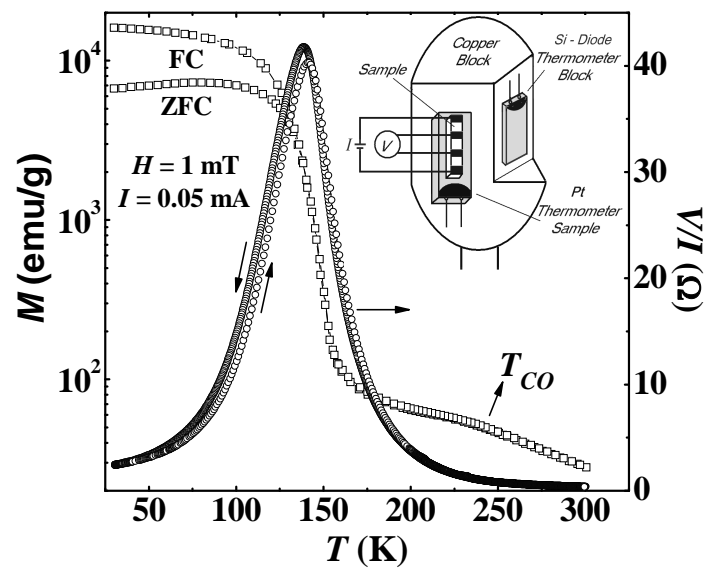

FIG. 1: $V / I(T)$ (right axis) and $M(T)$ (left axis) of $\mathrm{Nd}_{0.5} \mathrm{Ca}_{0.5} \mathrm{Mn}_{0.96} \mathrm{Cr}_{0.04} \mathrm{O}_{3}$. The inset displays the copper block sample holder experimental setup. Warming and cooling cycles in $V / I(T)$ measurements $(I=0.05 \mathrm{~mA})$ are indicated by arrows.

We report the occurrence of abrupt jumps/drops on the $V$ across the phase-separated manganite 
$\mathrm{Nd}_{0.5} \mathrm{Ca}_{0.5} \mathrm{Mn}_{0.96} \mathrm{Cr}_{0.04} \mathrm{O}_{3}$ (Cr-NCMO). By using an appropriated experimental setup we have observed that the occurrence of nonlinearity in $I-V$ curves is a precursor of a drastic rise in the temperature of the sample $T_{S}$. A model to account for the increase in $T_{S}$ on increasing $I$ supports the experimental data. Moreover, based on the temperature dependence of the specific heat $C_{p}$, an estimate of the energy dissipation of the material further confirms the importance of Joule heating effects. The results allowed us to propose that Joule self-heating effects associated with localized conduction are essential ingredients to understand the abrupt $I$-induced phase transition in phase-separated manganites.

Polycrystalline samples of Cr-NCMO were prepared by solid state reaction 14 Four-wire dc $\rho(T)$ and $I-V$ measurements were performed between 30 and $300 \mathrm{~K}$. Four gold contact pads were deposited on parallelepipedshaped (typically of length $l=0.60 \mathrm{~cm}$, width $w=0.145$ $\mathrm{cm}$, and thickness $t=0.075 \mathrm{~cm}$ ) samples to obtain excellent electric contacts by using Ag epoxy. In $I-V$ measurements, the current sweeps between $-100 \leq I \leq 100 \mathrm{~mA}$ were carried out in $0.5 \mathrm{~mA}$ steps. To avoid any memory effects, after each measurement the sample was heated to $300 \mathrm{~K}$, cooled down to $30 \mathrm{~K}$, and then heated to the desired measuring $T$. The experimental setup for the electrical measurements uses a cold finger connected to a closedcycle helium refrigerator. The temperature of the system $T$ is monitored with a silicon diode, positioned near the sample, and mounted on the copper block. The temperature of the sample $T_{S}$ is measured by a $\mathrm{Pt}$ thermometer placed on the copper block. The sample stands over the Pt thermometer and is fixed to it by using a thin thermal conducting silicon grease layer. The inset in Fig [ displays a schematic draw of the sample holder setup. Magnetization $M(T)$ measurements were performed in a SQUID magnetometer under applied magnetic field of $1 \mathrm{mT}$ in both FC and ZFC modes. $C_{p}$ measurements in Cr-NCMO crystals were performed in a Quantum Design PPMS apparatus.

The compound $\mathrm{Nd}_{0.5} \mathrm{Ca}_{0.5} \mathrm{MnO}_{3}$ has a $\mathrm{CO}$ antiferromagnetic-insulator (AFI) ground state below $T_{C O} \sim 240 \mathrm{~K}$ where a partial $\mathrm{OO}$ and magnetic correlations of short range are observed 1.15 At low $T$, the $\mathrm{OO}$ increases and a long range CE-type AFI state is established below $T_{N} \sim 170 \mathrm{~K}$. Substitutions on the Mn site are an effective way to gradually modify the $\mathrm{CO}$ state 1 The material under investigation $\mathrm{Nd}_{0.5} \mathrm{Ca}_{0.5} \mathrm{Mn}_{0.96} \mathrm{Cr}_{0.04} \mathrm{O}_{3}$ is well studied and develops a FMM phase at low temperatures, as shown in Fig [1.15 The transition to the CO regime is inferred from a subtle change of $d \rho / d T$ and a small cusp in the $M(T)$ data at $T_{C O} \sim 240 \mathrm{~K} \stackrel{1.15}{=}$ With decreasing $T$, a MI transition, closely related to the FM transition, occurs at $T_{M I} \sim 140$ $\mathrm{K}$ when $I=0.05 \mathrm{~mA}$. The thermal irreversibility in both $M(T)$ and $\rho(T)$ data indicate a coexistence of $\mathrm{CO} / \mathrm{OO}$ and FMM phases at low temperatures ${ }^{14.16}$ Indeed, under Cr-doping and for $T<T_{M I}$, these compounds are comprised of a fine mixture of $20-30 \mathrm{~nm}$ domains of the

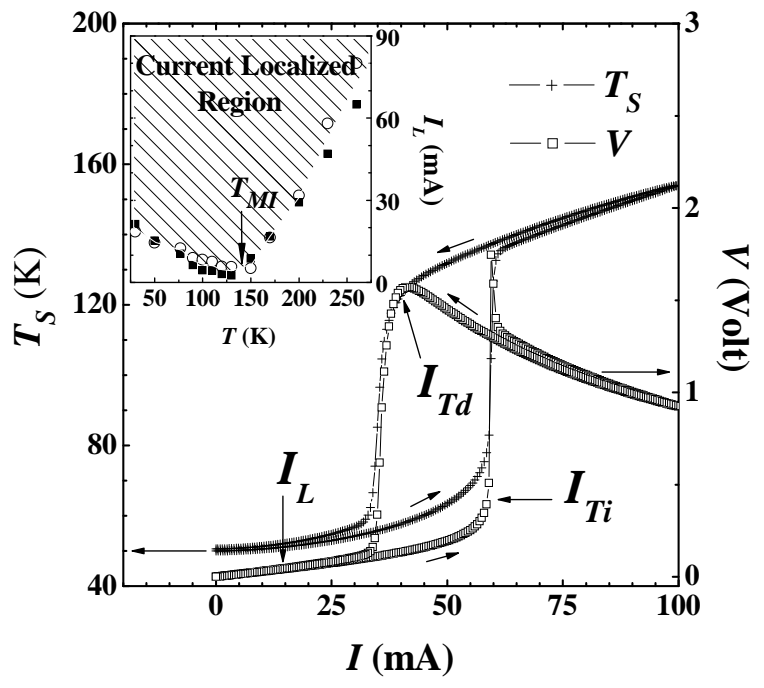

FIG. 2: $I-V$ curve at $50 \mathrm{~K}$ (right, open squares) and the corresponding temperature rise of the sample $T_{S}$ (left, crosses) vs. $I$. The inset shows the temperature dependence of $I_{L}$. Arrows denote increasing and decreasing $I$.

FMM phase embedded in the $\mathrm{CO} / \mathrm{OO}$ matrix ${ }^{16}$

Fig 2 shows a typical $I$ - $V$ curve $(T=50 \mathrm{~K})$ and the temperature rise of the sample $T_{S}$ as $I$ increases. The data reveal a clear correspondence of both $V$ and $T_{S}$ curves against $I$. At low $I$, the rising in $T_{S}$ is smooth and the $I$ $V$ data is here considered linear up to an $I$ value $I_{L} \sim 14.5$ $\mathrm{mA} . I_{L}$ is defined as the current in which the temperature measured by the Pt-thermometer increases $1 \mathrm{~K}$. As $I$ evolves, the $I-V$ data loose the linearity and $T_{S}$ rises progressively. Further rising in $I$ leads to a switch to a much higher $V / I$ value, as inferred from a steep increase in $V$ at $I_{T i} \sim 59 \mathrm{~mA}\left(\sim 5.4 \mathrm{~A} / \mathrm{cm}^{2}\right)$. This $I$-induced phase transition is accompanied by a remarkable increase in $T_{S}$ from $\sim 70$ to $\sim 135 \mathrm{~K}$ in a narrow range of $I$ while $V$ displays a spike-like maximum. With further increasing in $I, V$ decreases monotonically, i. e., the $I$ - $V$ curves exhibit a negative differential resistance $(d V / d I<0)$ up to $I=100 \mathrm{~mA}^{6}$ This feature is also reproduced in $T_{S}$ that rises continuously, reaching $\sim 155 \mathrm{~K}$ for $I=100 \mathrm{~mA}$, while the temperature of the copper block varied $\sim 1.6 \mathrm{~K}$.

The $I$-induced phase transition at $I_{T i}$ is useful to separate two well defined regions in dynamical $I$ - $V$ curves: below and above $I_{T i}$, where the material can be considered as a metal or insulator, respectively. The decreasing branch of $I-V$ curves shows a pronounced irreversibility in $I$, preserving the more insulating state down to a lower value of the threshold current $I_{T d}(T=50 \mathrm{~K}) \sim 40 \mathrm{~mA}$. At this excitation current, $V$ drops rapidly, and the initial $I-V$ curve is recovered with further decreasing $I$. Again, $T_{S}$ follows essentially the same trend: it decreases from $T_{S} \sim 150 \mathrm{~K}$ but it is kept above $100 \mathrm{~K}$ while the more insulating state is preserved. Accordingly, close to $I_{T d}(T=50$ $\mathrm{K}) \sim 40 \mathrm{~mA}, T_{S}$ drops rapidly to $\sim 55 \mathrm{~K}$ and continuously decreases towards to the initial value $(50 \mathrm{~K})$.

Due to a thin conducting grease layer separating the 


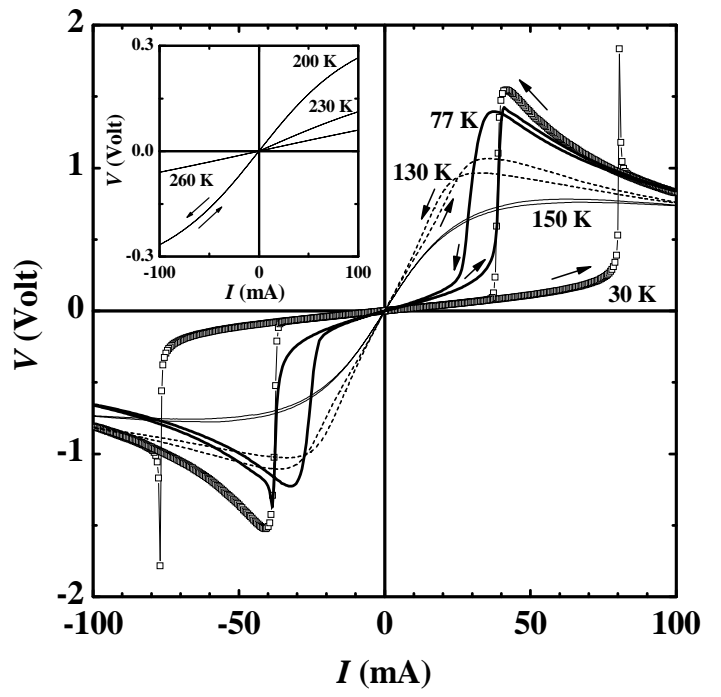

FIG. 3: $I-V$ curves of the Cr-NCMO compound at several temperatures. The inset displays the data in the vicinity of $T_{C O}$. The arrows point to the increasing and decreasing values of $I$.

Pt-thermometer and the sample, a temperature gradient $T_{G}$ in this region is expected to occur. An estimate of $T_{G}$ is made by comparing values of $V / I$ in $I$ $V$ curves and considering the $V / I$ vs. $T$ data of Fig 1 From Fig 2, the spike-like maximum in $V / I \sim 29 \Omega(I \sim 60$ $\mathrm{mA}$ ) corresponds to an average temperature reached by the sample of $\sim 152 \mathrm{~K}$ (see Fig प), a value compatible with the transition from metallic to insulating behavior but slightly higher than $T_{S} \sim 135 \mathrm{~K}$ measured by the Ptthermometer. Such a difference $T_{G} \sim 17 \mathrm{~K}$ is certainly related to the thermal coupling between sample and the Pt-thermometer but not only. A temperature gradient between the sample surface and the bottom is also expected since the thermal conductance in manganites is rather low 17 In addition, the estimated average sample temperature extracted from the $V / I$ data of Fig 1 taken at constant and rather low value of $I=0.05 \mathrm{~mA}$, must be also considered. For such low values of $I$ a homogeneous distribution of $I$ within the sample is expect, a feature hardly believed to occur at $I \sim 60 \mathrm{~mA}$.

A set of $I-V$ curves taken at different $T$ is displayed in Fig 3 The most prominent feature here is the sharp jump in $V$ at $I \sim I_{T i}$, which decreases appreciably with increasing $T$ and is barely identified for $T>T_{M I}$ (inset of Fig 3). Increasing $T$ also results in a less pronounced irreversibility of the $I-V$ curves for $T<T_{M I}$ and, for $T>T_{M I}$, they exhibit a reversible behavior 18 This indicates that the irreversibility in $I-V$ curves is close related to the phase competition in the phase-separated manganite, being larger when the FMM phase is robust, and further evidences the localized electric conduction in the FMM phase. The sweep to negative $I$ values leads to an antisymmetric $I-V$ behavior of the curves.

From $I$ - $V$ curves at different $T$, values of $I_{L}$ were computed and are shown in the inset of Fig 2 Such a dia- gram is useful for separating regions where current localization takes place in these materials. The decrease of $I_{L}$ with increasing $T \leq T_{M I}$ reflects the reduction of the relative volume fraction (VF) of the FMM phase, which coexist with the CO/AFI ${ }^{1.14 .16}$ Such a reduction is consistent with a more localized $I$ distribution across the material and provides an explanation for the so-called $I$ induced change in $T_{M I} \stackrel{19.20}{ } \mathrm{~A}$ considerable increase of $I_{L}$ for $T>T_{M I}$ suggests a much more homogenous distribution of $I$ across the sample in the insulating phase ${ }^{13,20}$ However, the current localization at high applied $I$ and $T>T_{M I}$ is probably related to the inhomogeneous nature of the CO-state, in which different structural phases were found to coexist 15.21 These observations suggest that both the temperature dependence of $I_{L}$ and the abrupt jump in $V$ are intimately related to the $\mathrm{VF}$ of the metallic phase within the material which is believed to vanish when the average temperature of the sample is $T \gtrsim T_{M I}$.

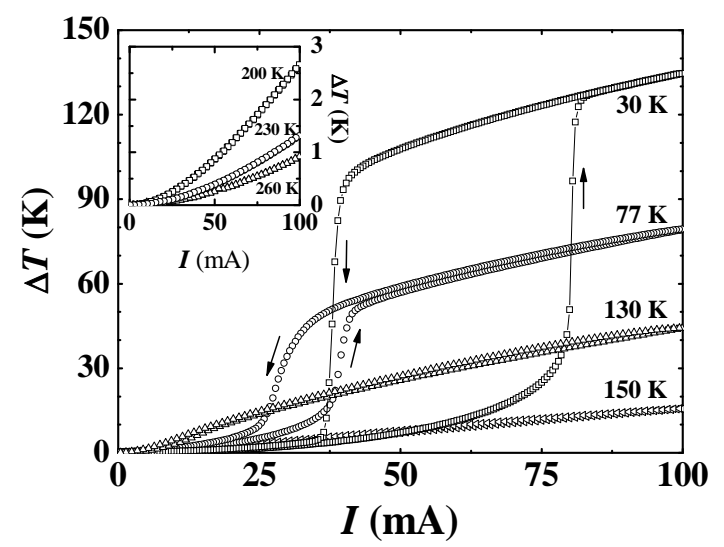

FIG. 4: Temperature rise of the sample $\Delta T=T_{S}-T$ vs. $I$. The copper-block sample holder measuring temperatures $T$ for each $I-V$ curve are indicated. The inset shows $\Delta T$ close to $T_{C O}$. The arrows point to the increasing and decreasing values of $I$.

We have carefully checked changes in the temperature of the copper block in all $I-V$ curves. The data show a maximum temperature rise of the block of $\sim 1.9 \mathrm{~K}$ at $T=30 \mathrm{~K}$, when the sample is subjected to $I=100 \mathrm{~mA}$ and $T_{S} \sim 165 \mathrm{~K}$. Thus, the temperature rise of the sample with respect to the copper block has been defined as $\Delta T=T_{S^{-}}$ $T$ and typical data of $\Delta T$ vs. $I$ are shown in Fig 4 At $T=30 \mathrm{~K}$, a huge rise of $\sim 100 \mathrm{~K}$ in $\Delta T$, at essentially the same $I \sim 80 \mathrm{~mA}$ where $V$ increases drastically, is observed. The average temperature reached by the sample $T_{S}$ just after the jump was measured to be $\sim 155 \mathrm{~K}$, a value higher than $T_{M I} \sim 140 \mathrm{~K}$. Such a rise in $T_{S}$ is certainly caused by Joule self-heating effects, a process that continues up to $I=100 \mathrm{~mA}$, where $T_{S} \sim 165 \mathrm{~K}$. When the sample was subjected to a power dissipation of $\sim 0.1 \mathrm{~W}(I=100 \mathrm{~mA})$, the highest value of $\Delta T \sim 135 \mathrm{~K}$ was observed for $T=30 \mathrm{~K}$. Again, a temperature gradient $T_{G} \sim 15 \mathrm{~K}$, at $I=100 \mathrm{~mA}$, was estimated, further indicating a current localization, as already mentioned. Such a $T_{G}$ of the experimental 
arrangement is more pronounced at high $I$ and $T \leq T_{M I}$, indicating that the local temperature of the sample is significantly altered by the current localization.

The behavior of $\Delta T$ also displays irreversibility in curves taken at low temperatures, a feature that vanishes for $T \geq T_{M I}$. Increasing $T$ results in smaller values of $\Delta T$, being $\Delta T \sim 2 \mathrm{~K}$ for $I=100 \mathrm{~mA}$ at $T \sim T_{C O}$ (inset of Fig (4). Therefore, the experimental data suggest that changes in $I-V$ curves are close related to Joule selfheating effects, to the VF of the FMM phase, and to the localized distribution of $I$ in the metallic phase.

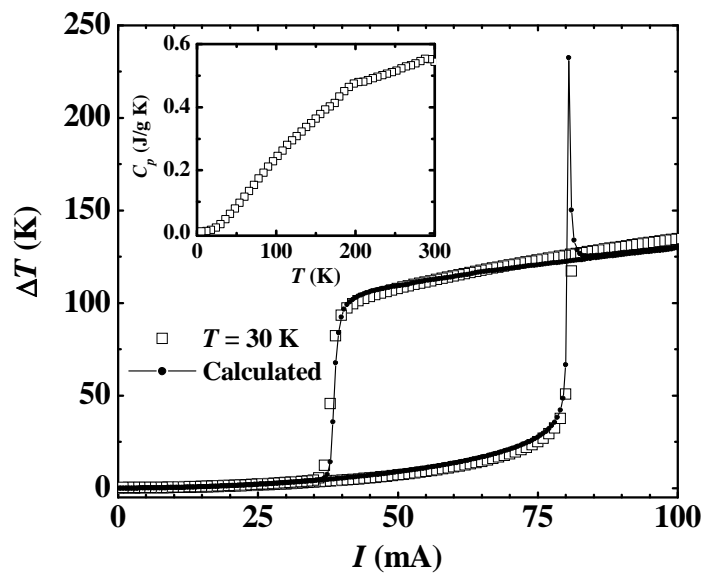

FIG. 5: The $I$ vs. $\Delta T$ curve at $30 \mathrm{~K}$ (open squares) and the calculated Joule-self heating effect (solid circles and line). The inset displays the $C_{p}(T)$ data of a single crystal specimen.

To support the experimental findings, the expected increase in $T_{S}$ due solely to Joule self-heating effects was calculated by considering the heat dissipation in the sample under applied $I$ and the heat conduction by the grease placed between the sample and the $\mathrm{Pt}$ thermometer ${ }^{22}$ Since $T$ was found to be nearly constant during the experiment, the temperature rise of the sample $\Delta T(T)$ can be approximated by $P / \kappa_{g}(A / d)$, where $P$ is the power dissipated in the sample, and $\kappa_{g}$ is the thermal conductivity of the grease with area $A$ and thickness $d$. By using the experimental $R=V / I$ data and $P=I^{2} \rho^{*}(l / w t)$, $\Delta T(T, I)=I^{2} \rho(T+\Delta T) / w t \kappa_{g}{ }^{*}(l d / A)$, where $w, l$, and $t$ were already defined. Considering that $\kappa_{g} \sim 0.16$ $\mathrm{Wm}^{-1} \mathrm{~K}^{-1}$ is nearly temperature independent in the $T$ range investigated $(30-160 \mathrm{~K}) \stackrel{23}{\stackrel{23}{2}}$ an example of the cal- culated $\Delta T(T=30 \mathrm{~K}, I)$ for a typical sample is shown in Fig [ The good agreement between the calculated and the measured $\Delta T(T)$ confirms that Joule self-heating provides an excellent description for the temperature rise of the sample. Small deviations in the high $I$ region are mainly related to: (i) the transition to the insulating regime where the VF of the FMM is low; (ii) changes in $T$ that is assumed to be constant; (iii) a thermal gradient within the sample; and (iv) a more homogeneous distribution of $I$ across the sample (see inset of Fig 22) ${ }^{5.13}$ Furthermore, the $C_{p}(T)$ data of a Cr-NCMO single crystal specimen (inset Fig 5) allowed us to estimate the energy required to heat the sample at a given temperature and to compare with the one due to Joule self-heating effects. By using the $C_{p}$ data, the energy required to heat a $\mathrm{Cr}$ NCMO sample from 30 to $140 \mathrm{~K}$ was estimated to be $\sim 85$ $\mathrm{mJ}$. Accordingly, the energy dissipated by $I_{T i}(T=30 \mathrm{~K})$ was found to be $\sim 80 \mathrm{~mJ}$, corresponding to a $T_{S} \sim 140$ $\mathrm{K}$, in excellent agreement with the data shown in Fig 5 . This result supports the experimental data, and lends credence to the relationship between Joule self-heating effects and the temperature rise of the sample.

In summary, the combined experimental results indicate that the abrupt increase of $T_{S}$ can be doubtless ascribed to the heat generated by the $I$ flow through localized pathways in the FMM phase. Under relatively low $I$, the weaker metallic regions become insulating, increasing the current density in the remaining FMM paths. ${ }^{\frac{5}{n}}$ This is reflected in both the initial rise of $T_{S}$ and changes in the $I$ - $V$ behavior. As $I$ evolves, the weaker metallic paths are progressively reduced and the Joule self-heating effect increases, culminating in a rapid collapse of the FMM phase ${ }^{\frac{5}{5}}$ This is mirrored in the observed jumps in $V$ and the dramatic rise of $T_{S}$. The Joule self-heating is strong enough to promote the sample to the insulating phase $\left(T_{S}>T_{M I}\right)$, resulting in a less appreciable self-heating due to a much more homogeneous distribution of $I \stackrel{13,20}{=}$ The negative differential resistance at high $I$ values (Figs.2 and 3$)$ is a consequence of the decrease in $V / I\left(T>T_{M I}\right)$ as $T_{S}$ still rises with increasing $I$.

This work was supported by the Brazilian agency FAPESP under Grant No. 99/10798-0, 01/01454-8, and 01/04231-0. R. F. J. and F. C. F. are CNPq (Brazil) fellows under Grant No. 303272/2004-0 and 301661/2004-9, respectively.
* Electronic address: rjardim@if.usp.br

1 T. Kimura et al., Phys. Rev. B 62, 15021 (2000).

2 Y. Moritomo et al., Phys. Rev. B 55, 7549 (1997).

3 K. Miyano et al., Phys. Rev. Lett. 78, 4257 (1997).

4 A. Asamitsu et al., Nature (London) 388, 50 (1997).

${ }^{5}$ M. Fiebig et al., Science 280, 1925 (1998).

6 A. Guha et al., Phys. Rev. B 62, 5320 (2000).

7 A. Guha et al., Appl. Phys. Lett. 75, 3381 (1999).

8 S. Mercone et al., Phys. Rev. B 65, 214428 (2002).
9 J. Stankiewicz et al., Phys. Rev. B 61, 11236 (2000).

10 Y. Tokura and N. Nagaosa, Science 288, 462 (2000).

11 K. Hatsuda et al., Appl. Phys. Lett. 83, 3329 (2003).

12 A. Wahl et al., Phys. Rev. B 68, 094429 (2003).

13 M. Tokunaga et al., Phys. Rev. Lett. 93, 037203 (2004).

14 A. S. Carneiro et al., J. Appl. Phys. 93, 8074 (2003).

15 W. Schuddinck et al., J. Magn. Magn. Mater. 211, 105 (2000).

16 S. Mori et al., Phys. Rev. B 67, 012403 (2003). 
17 J. L. Cohn et al., Phys. Rev. B 56, R8495 (1997).

18 The irreversible behavior of both $V$ and $T_{S}$ vs. $I$ curves below $T_{M I}$ are further evidence of the phase-separation in this manganite. In this $T$ range both $I_{T i}$ and $I_{T d}$, along with the difference $I_{T i}-I_{T d}$, decrease with increasing $T$ and approach zero at $T \sim T_{M I}$.

19 F. X. Hu and J. Gao, Phys. Rev. B 69, 212413 (2004).
20 A. Palanisami et al., Phys. Rev. B 71, 094419 (2005).

21 See, for example: J. Xu et al., Physica C 357-360, 401 (2001); S. Xu et al., J. Phys. Soc. Jpn. 72, 922 (2003); Y. Moritomo et al., ibid. 71, 1626 (2002).

22 P. Padhan et al., Phys. Rev. B 70, 134403 (2004).

${ }^{23}$ L. C. Sim et al., Thermochim. Acta 430, 155 (2005). 